\title{
CASE REPORT OF BUERGER'S DISEASE WITH GANGRENOUS GALL BLADDER AND SMALL GUT
}

\author{
Manju Singh ${ }^{1}$, Gambheer Singh², Amit Agrawal ${ }^{3}$, Sandeep Chandrakar ${ }^{4}$
}

\section{HOW TO CITE THIS ARTICLE:}

Manju Singh, Gambheer Singh, Amit Agrawal, Sandeep Chandrakar. "Case Report of Buerger's Disease with Gangrenous Gall Bladder and Small Gut". Journal of Evolution of Medical and Dental Sciences 2014; Vol. 3, Issue 55, October 23; Page: 12676-12682, DOI: 10.14260/jemds/2014/3679

ABSTRACT: Gangrenous gall bladder in Buerger's Disease is a rare presentation and has not been reported in past before in the literature. We present a case of intestinal obstruction in a 50 year male patient of Buerger's disease which contained gangrenous gall bladder. An exploratory laparotomy was done, resection of gangrenous small intestine 4 feet from Duodenojejunal junction up to 1 foot from Ileocecal junction with cholecystectomy and jejunostomy was done.

KEYWORDS: Gangrenous gall bladder, buerger's disease, rare case.

INTRODUCTION: Thromboangiitis Obliterans (Buerger's disease) is a non-atherosclerotic segmental inflammatory disease that most commonly affects the small and medium-sized arteries, veins, and accompanying nerves of the arms and legs. We are presenting a unique case of Buerger's disease with gangrenous gall bladder along with intestinal obstruction with gangrenous small intestine. No such case is being reported previously in the literature.

CASE REPORT: A 50 year male known case of Burgers disease presented in the emergency department of DR. B.R.A.M Hospital, Raipur on 13/11/2010 with complaints of severe abdominal pain and obstipation since 2 days. He had past history of chemical sympathectomy and he was a chronic smoker.

On admission, he was having poor general condition, tachycardia and hypotension. His abdomen was tense, distended and tender. There was no guarding and rigidity. His bowel loops were visible and bowel sounds were sluggish in all quadrants. His abdominal radiograph showed dilated bowel loops with multiple air fluid levels. His chest radiograph was normal. Ultrasonography of abdomen showed gallbladder sludge, minimal anechoic collection in Morrison's pouch and inters bowel area, few thickened bowel loops seen in right iliac fossa with no peristaltic movement.

Thickening of bowel loops was approximately $4.5 \mathrm{~mm}$ suggestive of infective pathology. On Arterial Colour Doppler of both lower limbs, the left anterior tibial, posterior tibial and dorsalis pedis artery showed sluggish colour flow and obstructive graph pattern. Rest of the arteries showed normal colour flow and graph pattern.

He was resuscitated and shifted to operation room with proper consent. Formal exploratory laparotomy was done. There was gangrenous jejunum and ileum 4 feet from Duodenojejunal junction up to 1 foot from Illeocecal junction. On further exploration there was distended, gangrenous gall bladder. 


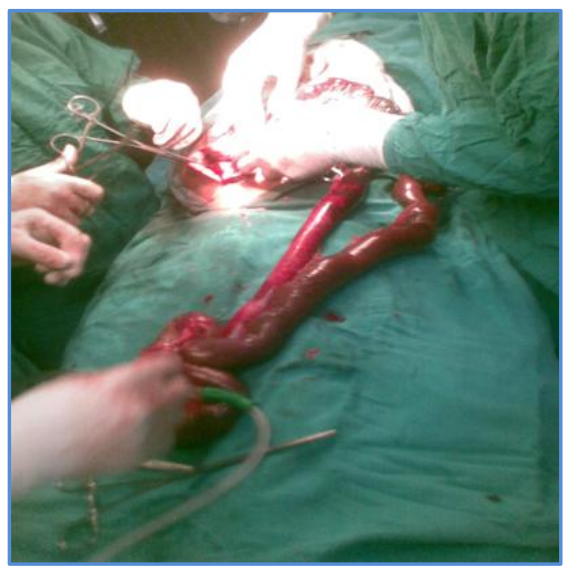

Fig. 1: Gangrenous small intestine

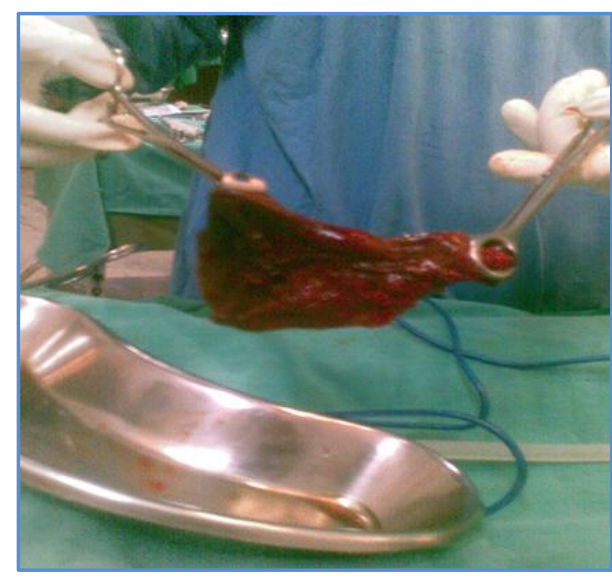

Fig. 2: Gangrenous gall bladder

The gangrenous intestine was resected, followed by cholecystectomy. Proximal end jejunostomy was made. The resected specimens were sent for histopathological examination. In postoperative period patient landed in sepsis and renal failure. His urea was $119 \mathrm{mg} / \mathrm{dl}$ and creatinine was $5.6 \mathrm{mg} / \mathrm{dl}$, associated with leukocyte count of 32,000/cu $\mathrm{mm}$ with $80 \%$, International Normalized Ratio of Prothrombin Time -2.27. Patient was aggressively treated in Surgical Intensive Care Unit by appropriate antibiotics, intravenous fluids and electrolytes corrections. Patient recovered and subsequently started on oral diet and discharged with properly functioning stoma on $18^{\text {th }}$ postoperative day.

DISCUSSION: Thromboangiitis Obliterans is a segmental non-atherosclerotic inflammatory disorder that involves primarily the small and medium sized arteries, veins, and nerves of the extremities. Von Winiwarter provided the first description of a patient with thromboangiitis obliterans in 1879.

Thromboangiitis obliterans is also known as Buerger's disease, named after Leo Buerger who published a detailed description of the pathological findings of amputated limbs in patients with the disease in 1908. Although it is observed worldwide, Thromboangiitis Obliterans is more prevalent in the Middle East and Far East. The disease typically presents in patients below 45 years of age. Young men are more frequently affected, but Thromboangiitis Obliterans also occurs in women.

RISK FACTORS: Exposure to tobacco is central to the initiation, maintenance, and progression of Thromboangiitis Obliterans. Although smoking tobacco is by far the most common risk factor, it may also develop as a result of chewing tobacco or marijuana use. Nearly two thirds of patients with Thromboangiitis Obliterans have severe periodontal disease and chronic anaerobic periodontal infection may represent an additional risk factor for the development of the disease. Polymerase chain reaction analysis demonstrated DNA fragments from anaerobic bacteria in both arterial lesions and oral cavities of patients with Thromboangiitis Obliterans but not in arterial samples from healthy control subjects.

PATHOPHYSIOLOGY: Thromboangiitis Obliterans is a vasculitis characterized by a highly cellular inflammatory thrombus with relative sparing of the vessel wall. Although acute-phase reactants such 
as erythrocyte sedimentation rate, C-reactive protein and commonly measured autoantibodies are typically normal, abnormalities in immune reactivity are believed to drive the inflammatory process. Patients with Thromboangiitis Obliterans have been shown to have increased cellular immunity to types I and III collagen compared with those who have atherosclerosis. ${ }^{3}$ In addition, high titers of ant endothelial cell antibodies have been detected in patients with this disorder. ${ }^{4}$

Prothrombotic and hemorheologic factors may also play a role in the pathophysiology of Thromboangiitis Obliterans. The prothrombin gene mutation $20210^{5}$ and the presence of anticardiolipin antibodies ${ }^{6}$ are associated with an increased risk of the disease. Thromboangiitis Obliterans patients with high anti cardiolipin antibody titers tend to have a younger age of onset and an increased rate of major amputation compared with patients who do not have detectable antibodies. ${ }^{6}$ Hemorheologic parameters such as hematocrit, red blood cell rigidity, and blood viscosity are increased in patients with Thromboangiitis Obliterans compared with those with atherosclerosis. $^{7}$

Thromboangiitis Obliterans involves 3 phases: acute, sub-acute, and chronic. The acute phase is composed of an occlusive, highly cellular, inflammatory thrombus. Polymorphonuclear leukocytes, micr abcesses, and multinucleated giant cells are often present. The chronic phase is characterized by organized thrombus and vascular fibrosis that may mimic atherosclerotic disease. However, Thromboangiitis Obliterans in any stage is distinguished from atherosclerosis and other vasculatures by the preservation of the internal elastic lamina.

CLINICAL PRESENTATION: Patients with Thromboangiitis Obliterans typically present with ischemic symptoms caused by stenosis or occlusion of the distal small arteries and veins. Involvement of both the upper and lower extremities and the size and location of affected vessels help distinguish it from atherosclerosis. Although symptoms may begin in the peripheral portion of a single limb, Thromboangiitis frequently progresses proximally and involves multiple extremities.

Arterial occlusive disease resulting from Thromboangiitis Obliterans often presents as intermittent claudication of the feet, legs, hands, or arms. Symptoms and signs of critical limb ischemia, including rest pain, ulcerations, and digital gangrene, occur with more advanced disease. Reynaud's phenomenon is present in $>40 \%$ of patients with Thromboangiitis Obliterans and may be asymmetrical. ${ }^{1}$ Although most common in the extremities, Thromboangitis Obliterans may also involve the cerebral, coronary, renal, mesenteric, and pulmonary arteries. ${ }^{1}$

Superficial thrombophlebitis differentiates Thromboangiitis Obliterans from other vasculatures and atherosclerosis, although it may also be observed in Behçet's disease. Superficial thrombophlebitis may predate the onset of ischemic symptoms caused by arterial occlusive disease and frequently parallels disease activity. Patients may describe a migratory pattern of tender nodules that follow a venous distribution.

The physical examination of a patient with suspected Thromboangiitis Obliterans includes a detailed vascular examination with palpation of peripheral pulses, auscultation for arterial bruits, and measurement of ankle: brachial pressure indices. The extremities should be inspected for superficial venous nodules and cords, and the feet and hands should be examined for evidence of ischemia. Although nonspecific, a positive Allen test in a young smoker with digital ischemia is strongly suggestive of the disease. Neurological examination may document peripheral nerve involvement, with sensory findings in up to $70 \%$ of patients. $^{1}$ 


\section{CASE REPORT}

DIAGNOSIS: Thromboangiitis Obliterans is a clinical diagnosis that requires a compatible history, supportive physical findings, and diagnostic vascular abnormalities on imaging studies. Several criteria have been proposed for the diagnosis of Thromboangiitis Obliterans. Common clinical criteria include age $<45$ years; current or recent history of tobacco use; distal extremity ischemia confirmed by noninvasive testing; exclusion of thrombophilia, autoimmune disease, diabetes, and a proximal source of emboli; and consistent angiographic findings. ${ }^{1}$

Laboratory testing in patients with suspected Thromboangiitis Obliterans is used to exclude alternative diagnoses. Initial laboratory studies should include a complete blood count, metabolic panel, liver function tests, fasting blood glucose, inflammatory markers such as erythrocyte sedimentation rate and C-reactive protein, cold agglutinins, and cryoglobulins. In addition, serological markers of autoimmune disease, including antinuclear antibody, ant centromere antibody, and antiSCL-70 antibody, should be obtained and are typically negative in Thromboangiitis Obliterans.

Lupus anticoagulant and anticardiolipin antibodies are detected in some patients with Thromboangiitis Obliterans but may also indicate an isolated thrombophilia. Echocardiography may be indicated in certain cases when acute arterial occlusion caused by thromboembolism is suspected to detect a cardiac source of embolism. Computed tomographic, magnetic resonance, or invasive contrast angiography may be performed to exclude a proximal arterial source of embolism and to define the anatomy and extent of disease.

Although advances in computed tomographic and magnetic resonance angiography show promise for imaging distal vessels, most patients require invasive contrast angiography to provide the spatial resolution necessary to detect small-artery pathology. Distal small- to medium-artery involvement, segmental occlusions, and "corkscrew"-shaped collaterals around areas of occlusion are typical angiographic findings in Thromboangiitis Obliterans. Proximal arteries should be normal without evidence of atherosclerosis. Biopsy is rarely indicated but is most likely to be diagnostic in a vein with superficial thrombophlebitis during the acute phase of the disease.

PROGNOSIS: The prognosis for patients with Thromboangiitis Obliterans depends largely on the ability to discontinue tobacco use. In a retrospective series of 110 patients with Thromboangiitis Obliterans, 43\% of patients underwent 108 amputation procedures. ${ }^{8}$ Among those who continued smoking, 19\% required a major amputation. None of those who stopped smoking underwent amputation. A substantial proportion (85\%) of patients with Thromboangiitis Obliterans who underwent major amputation lost their jobs.

MANAGEMENT: Discontinuation of tobacco use is the definitive therapy for Thromboangiitis Obliterans. Complete smoking cessation is essential because even a few cigarettes a day may result in disease progression. Patient education on the role of tobacco exposure in the initiation, maintenance, and progression of the Thromboangiitis Obliterans is paramount. Adjunctive measures to help patients discontinue tobacco use such as pharmacotherapy and smoking cessation groups should be offered.

Nicotine replacement therapy should be avoided because it may contribute to disease activity. Although patients with Thromboangiitis Obliterans are thought to have a greater degree of tobacco dependence than those with coronary atherosclerosis, no significant difference in time to tobacco cessation after diagnosis has been demonstrated. ${ }^{9}$ 
Surgical revascularization is usually not feasible in patients with Thromboangiitis Obliterans because of the distal and diffuse nature of the disease. However, bypass surgery may be considered in select patients with severe ischemia and suitable distal target vessels. Thromboangiitis Obliterans patients undergoing bypass surgery often have suboptimal outcomes with primary patency rates of $41 \%, 32 \%$, and $30 \%$ and secondary patency rates of $54 \%, 47 \%$, and $39 \%$ at 1,5 , and 10 years. ${ }^{8} \mathrm{Graft}$ patency rates are nearly $50 \%$ lower in patients with Thromboangiitis Obliterans who continue to smoke after surgery. ${ }^{10}$

Additional therapeutic options for the treatment of Thromboangiitis Obliterans have been limited to vasodilators, intermittent pneumatic compression, spinal cord stimulation, and peripheral periarterial sympathectomy. In a randomized controlled trial of 152 patients with the disease, patients treated with the prostanoid vasodilator iloprost had significant relief of rest pain, greater healing of ischemic ulcers, and a two-thirds reduction in the need for amputation. ${ }^{11}$ Other vasodilators such as $\alpha$-blockers, calcium channel blockers, and sildenafil may be helpful but have not been studied in prospective clinical trials.

Intermittent pneumatic compression of the foot and calves has been used to augment perfusion to the lower extremities in patients with severe claudication or critical limb ischemia who are not revascularization candidates because of distal arterial occlusive disease, including Thromboangiitis obliterans ${ }^{12}$.Epidural spinal cord stimulation has been shown to improve regional perfusion in patients with Thromboangiitis obliterans ${ }^{13}$.Peripheral periarterial sympathectomy is occasionally considered for patients with refractory pain and digital ischemia but remains controversial.

The limited options for patients with severe distal peripheral artery disease and critical limb ischemia have driven a growing interest in therapeutic angiogenesis. In a small study of patients with Thromboangiitis Obliterans, intramuscularly administered vascular endothelial growth factor resulted in the healing of ischemic ulcers and relief of rest pain. ${ }^{14}$ Several studies have evaluated autologous bone marrow mononuclear cell implantation for patients with critical limb ischemia resulting from Thromboangiitis obliterans. ${ }^{15-17}$ although short-term results of therapeutic angiogenesis have been promising, long-term safety and efficacy require further investigation. ${ }^{18}$

\section{REFERENCES:}

1. Olin JW. Thromboangiitis obliterans (Buerger's disease). N Engl J Med.2000; 343: 864-869.

2. Iwai $T$, Inoue $Y$, Umeda $M$, Huang $Y$, Kurihara N, Koike M, Ishikawa I. Oral bacteria in the occluded arteries of patients with Buerger disease. J Vasc Surg. 2005; 42: 107-115.

3. Adar R, Papa MZ, Halpern Z, Mozes M, Shoshan S, Sofer B, Zinger H, Dayan M, Mozes E. Cellular sensitivity to collagen in thromboangiitis obliterans. N Engl J Med. 1983; 308: 1113-1116.

4. Eichhorn J, Sima D, Lindschau C, Turowski A, Schmidt H, Schneider W, Haller H, Luft FC. Antiendothelial cell antibodies in thromboangiitis obliterans. Am J Med Sci. 1998; 315: 17-23.

5. Avcu F, Akar E, Demirkilic U, Yilmaz E, Akar N, Yalcin A. The role of prothrombotic mutations in patients with Buerger's disease. Thromb Res. 2000; 100: 143-147.

6. Maslowski L, McBane R, Alexewicz P, Wysokinski WE. Antiphospholipid antibodies in thromboangiitis obliterans. Vasc Med. 2002; 7: 259-264.

7. Bozkurt AK, Koksal C, Ercan M. The altered hemorheologic parameters in thromboangiitis obliterans: a new insight. Clin Appl Thromb Hemost. 2004; 10: 45-50. 


\section{CASE REPORT}

8. Ohta T, Ishioashi H, Hosaka M, Sugimoto I. Clinical and social consequences of Buerger disease. J Vasc Surg. 2004; 39: 176-180.

9. Cooper LT, Henderson SS, Ballman KV, Offord KP, Tse TS, Holmes DR, Hurt RD. A prospective, case-control study of tobacco dependence in thromboangiitis obliterans (Buerger's disease). Angiology. 2006; 57: 73-78.

10. Sasajima T, Kubo Y, Inaba M, Goh K, Azuma N. Role of infrainguinal bypass in Buerger's disease: an eighteen-year experience. Eur J Vasc Endovasc Surg.1997; 13: 186-192.

11. Fiessinger JN, Schafer M. Trial of iloprost versus aspirin treatment for critical limb ischaemia of thromboangiitis obliterans: the TAO Study. Lancet.1990; 335: 555-557.

12. Montori VM, Kavros SJ, Walsh EE, Rooke TW. Intermittent compression pump for nonhealing wounds in patients with limb ischemia: the Mayo Clinic experience (1998-2000). Int Angiol. 2002; 21: 360-366.

13. Donas KP, Schulte S, Ktenidis K, Horsch S. The role of epidural spinal cord stimulation in the treatment of Buerger's disease. J Vasc Surg. 2005; 41: 830-836.

14. Isner JM, Baumgartner I, Rauh G, Schainfeld R, Blair R, Manor O, Razvi S, Symes JF. Treatment of thromboangiitis obliterans (Buerger's disease) by intramuscular gene transfer of vascular endothelial growth factor: preliminary clinical results. J Vasc Surg. 1998; 28: 964-973.

15. Saito S, Nishikawa K, Obata H, Goto F. Autologous bone marrow transplantation and hyperbaric oxygen therapy for patients with thromboangiitis obliterans. Angiology. 2007; 58: 429-434.

16. Matoba S, Tatsumi T, Murohara T, Imaizumi T, Katsuda Y, Ito M, Saito Y, Uemura S, Suzuki H, Fukumoto S, Yamamoto Y, Onodera R, Teramukai S, Fukushima M, Matsubara H. Long-term clinical outcome after intramuscular implantation of bone marrow mononuclear cells (Therapeutic Angiogenesis by Cell Transplantation [TACT] trial) in patients with chronic limb is chemia. Am Heart J. 2008; 156: 1010-1018.

17. Durdu S, Akar AR, Arat M, Sancak T, Eren NT, Ozyurda U. Autologous bone-marrow mononuclear cell implantation for patients with Rutherford grade II-III thromboangiitis obliterans. J Vasc Surg. 2006; 44: 732-739.

18. Miyamoto K, Nishigami K, Nagaya N, Akutsu K, Chiku M, Kamei M, Soma T, Miyata S, Higashi M, Tanaka R, Nakatani T, Nonogi H, Takeshita S. Unblinded pilot study of autologous transplantation of bone marrow mononuclear cells in patients with thromboangiitis obliterans. Circulation. 2006; 114: 2679-2684. 


\section{AUTHORS:}

1. Manju Singh

2. Gambheer Singh

3. Amit Agrawal

4. Sandeep Chandrakar

\section{PARTICULARS OF CONTRIBUTORS:}

1. Associate Professor, Department of General Surgery, Pt. JNM Medical College, Raipur.

2. Managing Director, Department of General Surgery, Samarpan Hospital, Raipur.

3. Assistant Professor, Department of General Surgery, Pt. JNM Medical College, Raipur.
4. Assistant Professor, General Surgery, Pt. JNM Medical College, Raipur.

\section{NAME ADDRESS EMAIL ID OF THE CORRESPONDING AUTHOR:}

Dr. Amit Agrawal, H.I.G C/72,

Shailendra Nagar, Raipur-492001, Chattisgarh.

Email: doc.amitagrawal@gmail.com

Date of Submission: 25/09/2014. Date of Peer Review: 26/09/2014. Date of Acceptance: 17/10/2014. Date of Publishing: 23/10/2014. 